\title{
A MODEL BASED SYSTEMS ENGINEERING PROCESSES DEPLOYMENT FRAMEWORK
}

\author{
Clémentine Cornu, Bernard Chiavassa \\ Eurocopter, ETZP, Aéroport International Marseille Provence 13725 Marignane Cedex - France \\ \{Clementine.Cornu, Bernard.Chiavassa\}@eurocopter.com \\ Vincent Chapurlat \\ LGI2P - Site EERIE de l'EMA, Parc Scientifique George Besse 30035 Nîmes Cedex 1 - France \\ Vincent.Chapurlat@mines-ales.fr \\ François Irigoin \\ Mines ParisTech - CRI, 35 rue Saint Honoré, 77305 Fontainebleau Cedex - France \\ Francois.Irigoin@mines-paristech.fr
}

Keywords: Model Based Systems Engineering, Systems Engineering Process, Graphical Modeling Framework, Interoperability.

\begin{abstract}
Systems Engineering is a tried and tested methodological approach to design and test new products. It acts as a model based engineering approach and promotes for this purpose a set of standardized collaborative processes, modelling languages and frameworks. The systems engineering processes imply many interactions and exchanges between resources. Nevertheless, currently there is no method guiding companies in the deployment of these processes adapted to meet their stakeholders' expectations. Particularly, interoperability abilities and capacities which are required at each level of the company and by each resource remain poorly addressed. The purpose of this paper is twofold: 1) to present an approach for deploying systems engineering processes taking into account the interoperability assessment of resources to guide their allocations; and 2) to identify and propose a dedicated software framework having to be developed in order to support this approach.
\end{abstract}

\section{INTRODUCTION}

The Systems Engineering (SE) approach is considered today as an efficient methodological and interdisciplinary approach. It promotes a set of processes required to design and test a new product or service (ISO/IEC, 2008) (INCOSE, 2010) since the organization and the behaviour of a company are often described under the form of a map of inter dependant processes. Let us remain that a process can be defined as "a logical sequence of tasks performed to achieve a particular objective. A process defines "WHAT" is to be done, without specifying "HOW" each task is performed" (Estefan, 2008).

Nevertheless, despite the fact that SE is a tried and tested methodology, it is still difficult for companies to apply it. First, there is no method available describing how to practically deploy SE within companies. Second, the resources (e.g. actors, teams, computers, machines, software, etc.) having to be used, involved and coordinated all along a SE process generally do not have the required abilities, capacities, and even facilities, to efficiently work together, share information and communicate during the SE process activities. This can be considered as an interoperability question which is not currently considered during the deployment. Let us remain that interoperability can be defined as the "ability of enterprises and entities within those enterprises to communicate and interact effectively"(ISO, 2010). It is then considered here as a key factor of SE processes deployment.

The purpose of our research work is to provide companies a method to help them preparing the deployment of SE processes tailored to meet all 
stakeholders' expectations, interoperability requirements and specific constraints linked to the nature and the strategic objectives of the company. This method promotes:

- A roadmap of all activities the company must perform to define and formalize the adequate processes to deploy,

- A set of reference models and examples clarifying for instance the definition of activities or the allocation of resources, guiding thus the deployment and the tailoring of the proposed processes to the company,

- A meta-model describing all concepts required during the modelling of the components and the way of working of the company,

- A specific assessment method to appraise the interoperability of resources and thus to help in their allocation.

This paper focuses on the practical implementation of the SE process to deploy. This implementation is based on a framework which is defined here as a consistent group of software tools supporting the four elements of the methodological approach presented above.

This article is structured as follows: after a short presentation of the proposed deployment approach performed in Section 2, Section 3 presents the specific needs for software framework induced by this approach and presents the retained solutions before concluding.

\section{OVERVIEW OF DEPLOYMENT ACTIVITIES}

The purpose of this section is to outline the main principles and steps of the proposed deployment approach for SE processes. After defining the team in charge of the deployment, the four main steps of the approach are: 1) Model ideal processes to deploy and their relationships, 2) Model existing processes and their relationships, 3) Specify the processes to deploy and 4) Define practical implementation.

\subsection{Model ideal processes to deploy and their relationships}

The goal is to provide a model describing the idealised company's vision of a (set of) SE process(es) highlighting what would be each SE process, what must be the appropriate organization to adopt and what resources are required for optimal functioning of the process(es) to deploy. At this stage, "ideal" means there is no point here of being influenced by the current break-down structure of the company or by the current allocation of tasks.

This stage requires firstly to select a reference document (e.g. (ISO/IEC, 2008)) providing all theoretical definitions of processes. Secondly, the deployment team models these ideal processes taking into consideration the global specificities of the company and business area. To this end, a set of modelling languages has to be selected and adopted following the principles of enterprise modelling (Vernadat, 1996). Thirdly, the team models for each SE process, the process in charge of managing it. Finally, a deployment process is defined for each $\{\mathrm{SE}$ process; management process $\}$ couple.

\subsection{Model the existing processes and their relationships}

The purpose is now to characterize and analyse the current company's organisation defined for the design of new products or services. During this analysis, no constraint is induced by the ideal model of SE processes to deploy. The existing activities and resources involved or that could be involved in the design of products or services are characterized and modelled as an AS-IS model. To perform this stage, the team must select the scope of the analysis ("Which project/program will be used as a basis?"), collect information, and then formalize this information by using the same formalism as the one used in stage 2.1 .

\subsection{Specify the processes to deploy}

A TO-BE model (Chapurlat \& Braesch, 2008) is then proposed mixing and merging when necessary the ideal process(es) models and the AS-IS model. The goal is to perceive significant gaps and thus highlights ways of improving the current organization. The TO-BE model is thus built to share the trade-offs found between the current and the ideal organizations. Figure 1 summarizes the detailed activities to be carried out during this step for each SE process to deploy but also for the relevant management processes having to be deployed at same time.

Among the activities described in this figure let us focus on the resources allocation which is done according to potential and effective interoperability assessments. Interoperability refers here to the capacities and abilities of one or two resources to collaborate efficiently from organisational, conceptual and technical points of view. 


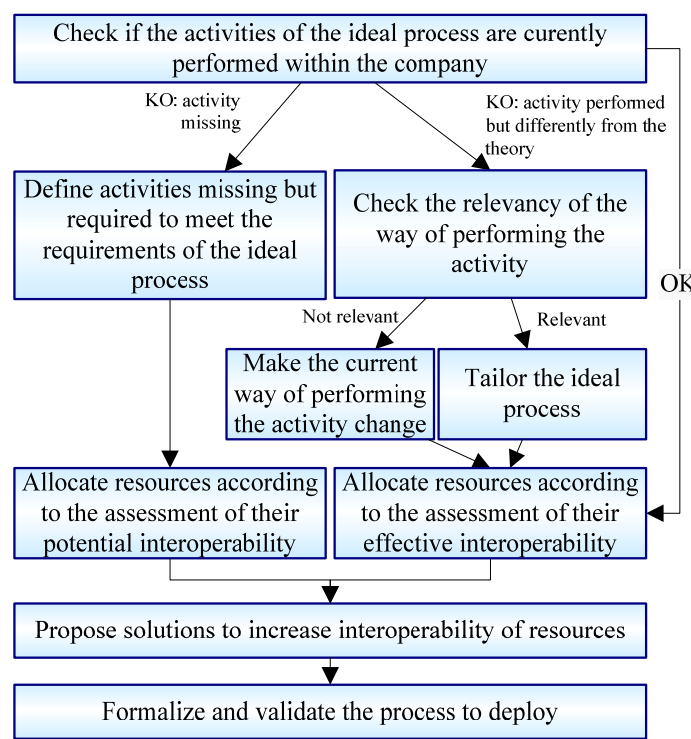

Figure 1: Final definition of processes to deploy

According to the needs of the deployment team, the appraisal is performed either on one single resource to assess its own abilities to interoperability; or on a couple of resources to especially assess their compatibility. Therefore, this appraisal is done either to define a current status of the ability of the resource(s) to be interoperable (effective interoperability); or to anticipate the future behaviour of resource(s) during new collaborations induced by the new organization provided by SE processes (potential interoperability). In this last case, the interoperability appraisal can be used by the deployment team for example to decide between two equivalent resources, or to detect interesting improvements on existing resource before any physical deployment.

\subsection{Define practical implementation}

Finally, the deployment team defines an action plan for the deployment addressing topics such as:

- the planning of the deployment,

- the organization of the communication about the deployment,

- the definition of the required training activities and the design of training materials,

- the definition of the transition phase: the mapping between old and new organization, roles and responsibility, way of working, tools, etc.

In order to support all activities for the preparation of the deployment, some software tools are required. Their specifications are provided in next section.

\section{FRAMEWORK SPECIFICATION}

Figure 2 provides an overview of the tools constituting the framework having to support the preparation of the deployment of SE processes. All theses tools are described in the following sections.

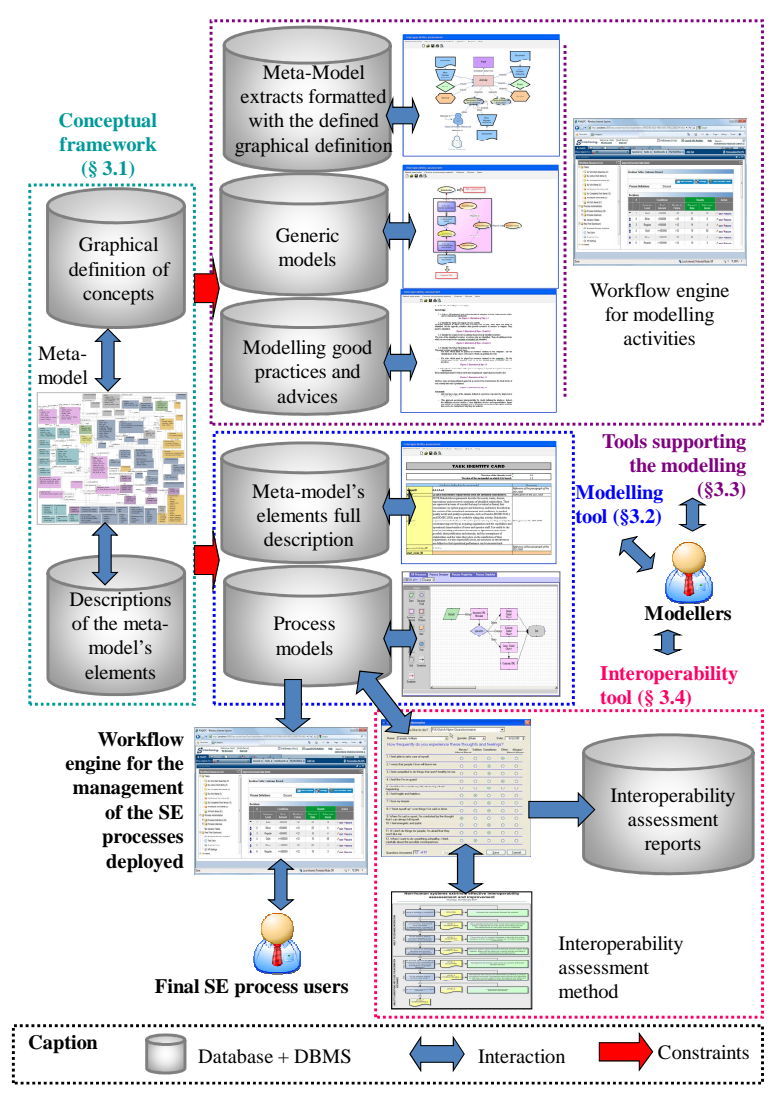

Figure 2: Overview of tools needed in the preparation of SE processes deployment

\subsection{Conceptual framework}

The deployment of SE processes requires a lot of concepts such as "resource", "process", "activity", "stakeholders", etc. They must be defined as soon as possible to facilitate and to guide the work of the deployment team. Indeed, the existence of a common repository of concepts and of relationships between concepts enables a common understanding between the team members. It supports the work to be done with all stakeholders involved in the deployment project, especially if they come from various business fields. By defining explicitly the concepts, their semantic relationships, the deployment team lessens then the risks of 
misunderstandings and thus increases the interoperability between deployment stakeholders.

A meta-model is proposed to describe all concepts, relationships, constraints and rules used to guide the steps described in Section 2 and particularly modelling activities. To illustrate our point, an extract of this meta-model is shown in Figure 3.

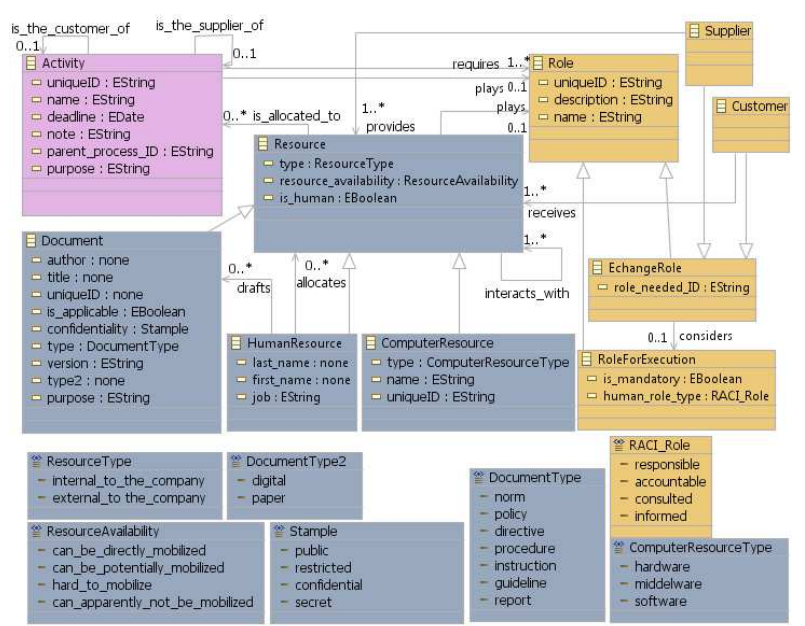

Figure 3: Example of meta-model (extract)

Nevertheless, this meta-model is not selfsufficient: it must be completed with a textual description of each class, attribute and relationship in order to help the understanding of the modeller. Furthermore, to ensure the syntactical consistency of the models built by the deployment team, a graphical representation of each class and relationship must also be defined.

All elements constituting the conceptual framework may evolve or be adapted according to the results of their application in a given company.

\subsection{Modelling tools}

The conceptual framework provides the basis for all modelling work. However, to support it, the deployment team needs a modelling tool including:

- a modelling workbench enabling to graphically represent the processes' components,

- a complementary tool for the description of models elements.

Therefore, among the various needs this modelling tool has to meet, we can mention:

N1: the modelling workbench must be able to read the meta-model and its graphical representation to constraint the building of models accordingly.
N2: the tool must be open-ended. Indeed, as said previously, the meta-model used as a basis is continuously improved as its application in company goes forward. So, any change in the conceptual framework must be performed quickly and must not induce instability of the modelling tool .

N3: for interoperability purpose, the tool must be "easily" connectable. Indeed, it must be compatible with other tools necessary for the deployment even if they are not known yet. Compatibility with the modelling tool currently used within the company must be considered.

N4: the tool must be able to support standard modelling languages such as the Unified Modeling Language (UML) or the Business Process Modeling Notation (BPMN) (OMG, 2011) (Wohed et al., 2006) to facilitate the understanding of any person who would read or annotate the model. Furthermore, the tool must be able to extend these modelling reference languages in order to add some required parameters such as those necessary for the interoperability appraisal.

N5: the models built with this tool must be in a format which facilitates the use of workflow engines for the management of the activities described in these models.

N6: the tool must be maintained at least during the design time. It is critical in the case of long-time design cycles such as those of aircrafts for example.

N7: the tool must be able to manage multiple versions of models and meta-model (configuration management).

N8: the tool must be able to add documentation about models and their elements.

All these needs induce difficulties to find a commercial off-the-shelf framework and tools which would be directly applicable and interoperable with the meta-model. The Graphical Modelling Framework (GMF) plug-in of Eclipse is proposed and its principles are illustrated on Figure 4.

Let us take back all desired functionalities to check the compliance of GMF:

N1: "the tool must use the defined metamodel and graphical definition". Considering the functioning of GMF, this need is met.

N2: "the tool must be open-ended". The functioning of GMF based on three separated layers enables easy changes and lessens the risk of loss of stability because of change.

N3: "the tool must be easily connectable". This need is met since GMF is based on Extensible Markup Language (XML) which is a standard generic language for exchanges. 


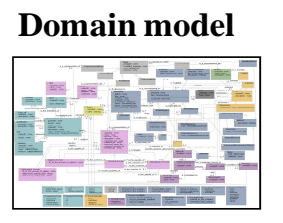

Meta-model: defined in xml or with a graphical editor
Graphical definition model

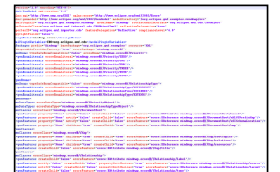

Graphical representation of the elements of domain model

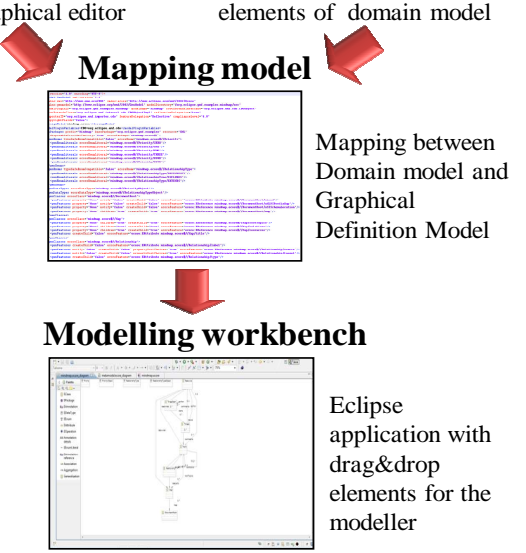

Figure 4: Basic elements constituting GMF

N4: "the tool must be able to support standard modelling languages". GMF supports any kind of modelling languages since the meta-model and the graphical definition for its elements are defined by the developer designing the modelling workbench.

N5: "the tool must facilitate the implementation of workflow engines". Here again, thanks to XML, this need is met.

N6: "the tool must be maintained at least during the design time". If the implementation of GMF is done by company's employees, it is up to the enterprise to manage the maintenance of the tool and to ensure that maintenance skills are available all over the design of its products.

N7: "the tool must manage versioning". This functionality is not performed by GMF.

N8: "the tool enables to add documentation about models and their elements". This functionality is included in GMF.

Consequently, only the need "N7" is not performed by GMF. However, this lack is not critical since in the worst case the versioning of models can at least be performed manually by the modeller.

\subsection{Tools supporting modelling activities}

We introduced previously two notions: the conceptual framework providing the set of concepts and rules for the modelling and the modelling tool providing required technical means. However, they both do not assist the modeller in his modelling work. Consequently, complementary tools have been added in this deployment framework.

\subsubsection{Tools organizing modelling activities}

During the preparation of the deployment, a lot of models must be built, probably by different modellers. Therefore, the framework for the deployment must include a tool helping to allocate, synchronize and monitor modelling activities. This can be easily done with a workflow engine chosen among those available on the market. Completing the selection criteria specific to the company, the following points can be considered.

First, for interoperability purpose, the tool must be "easily" connectable in order to be compatible with any other tools necessary for the deployment even if they are not known yet.

Then, as a workflow engine can also be used to manage deployed processes, we recommend using the same tool in the two contexts to limit the number of tools and thus the induced costs (licences, training, etc.). Therefore, needs for the workflow engine of the deployed processes must be considered when choosing the one for modelling activities. An example of these needs is the ability to communicate with technical tools used to performed SE activities (such as requirements management tools, design architecture tools, etc.) in order to collect and share the documents generated by them.

Finally, the possibility to use flexible workflows rather than traditional workflows must be envisaged. Indeed, contrary to traditional workflows where the execution of the sequence offers no freedom and where data are shared with difficulty, in flexible workflows, activities can be anticipated and collaborative work is increased thanks to shared data (Grigori et al., 2000). To this end, easiness of translations from BPMN to the Business Process Execution Language (BPEL) (OASIS, 2007)(White, 2005) must be considered.

\subsubsection{Tools facilitating modelling activities}

To assist the team members in their modelling activities, we have introduced in our framework tools providing them with:

- partial views of the meta-model with only the concepts and relationships they need at a given time and formatted with the up-to-date defined graphical representation of concepts.

- generic models such as models of processes, resources, or resources' roles since reuse or instantiation of models is time-saving. 
- modelling good practices and advices collected notably from enterprise modelling.

The methodological aspects of these tools are currently under development.

\subsection{Tool for the interoperability appraisal}

As presented in section 2, in order to define the SE processes to deploy, the appraisal of the resource abilities and capacities in terms of interoperability must be realized notably to decide between two equivalent resources.

To highlight the desired functionalities of the tool for the interoperability assessment, let us consider a simplified operational scenario

During its start-up, the tool loads from existing process models, the resources already modelled. Among them, the user selects the one(s) he needs and selects the kind of assessment he wants to use. If the tool does not have all information required to assess the resource(s), it asks the user for complementary information according to a questions tree defined in the interoperability assessment methodology. Once all data have been collected, the tool adds them to a local version of the process model which is submitted to the validation of the person in charge of the model. The tool generates a report to the user containing the details of the assessment, the analysis of these results and advices to improve the interoperability of the resource(s). A copy of this report is archived. Finally, if the person in charge of the model validates the modification proposed by the tool, the new version of the process model is included in the models database and the previous one is archived. If the modeller disagrees with the modifications proposed by the tool he can either achieve the necessary changes or reject the propositions.

A tool such as described in the operational scenario does not exist and has thus to be developed to meet the deployment team's needs. Like for the other tools, it must be open-ended and easily connectable. It must also be able to detect if the user has entered unexpected data.

\section{CONCLUSION AND FUTURE WORK}

In this paper, we present an innovative approach to deploy systems engineering processes. Its originality is the fact that it is based on models, enabling thus to build a bridge between systems engineering and enterprise modelling. This is a turnkey approach delivered with a full software framework designed to assist the team in charge of the deployment. It includes all technical means needed to build the required models but also a tool enabling to appraise the interoperability capability of the resources to be involved in the new process to deploy. The strength of this framework is its design thought to have a set of consistent, interoperable and open-ended tools limiting evolution problems and compatibility problems with tools already existing in the company. Thus, this equipped approach is a contribution to the introduction of systems engineering in companies dealing with the three classical dimensions of interoperability: conceptual, organisational, and technical.

\section{REFERENCES}

Chapurlat, V., \& Braesch, C., 2008. Verification, validation, qualification and certification of enterprise models: Statements and opportunities. Computers in Industry, 59(7), 711 - 721. Enterprise Integration and Interoperability in Manufacturing Systems.

Estefan, J., 2008. Survey of Model-Based Systems Engineering (MBSE) Methodologies. Tech. rept. INCOSE

Grigori, D., Skaf-Molli, H., \& Charoy, F. 2000. Adding flexibility in a cooperative workflow execution engine. Pages 227-236 of: 8th International Conference on High Performance Computing and Networking Europe - HPCN Europe 2000, Amsterdam, Hollande. Springer.

INCOSE, 2010. Systems Engineering Handbook - A guide for system life cycle processes and activities - v3.2.

ISO, 2010. ISO/DIS 11354-1 - Advanced automation technologies and their applications - Part 1: Framework for enterprise interoperability.

ISO/IEC, 2008. ISO/IEC 15288:2008 - Systems engineering - System life cycle processes.

OASIS, 2007. Web Services Business Process Execution Language Version 2.0. Tech. rept.

OMG, 2011. Business Process Model and Notation $(B P M N)$ Version 2.0. Tech. rept.

Vernadat, F., 1996. Enterprise modeling and integration: principles and applications. Kluwer Academic Publishers.

White, Stephen A., 2005. Mapping BPMN to BPEL Example. Tech. rept. IBM.

Wohed, P., Dumas, M., Hofstede, A. H. M. Ter, \& Russell, N., 2006. On the Suitability of BPMN for Business Process Modelling. Pages 161-176 of: In Proceedings 4th International Conference on Business Process Management (BPM 2006), LNCS. Springer Verlag. 